\title{
REVISITING THE SAMPLE PATH OF BROWNIAN MOTION
}

\author{
S. JAMES TAYLOR \\ 4, Knole Way, Sevenoaks, Kent \\ UK TN13 3RS \\ E-mail:sjt4k@aol.com
}

\begin{abstract}
Brownian motion is the most studied of all stochastic processes; it is also the basis for stochastic analysis developed in the second half of the 20th century. The fine properties of the sample path of a Brownian motion have been carefully studied, starting with the fundamental work of Paul Lévy who also considered more general processes with independent increments and extended the Brownian motion results to this class. Lévy showed that a Brownian path in $d(d \geq 2)$ dimensions had zero Lebesgue measure; he asked for the right Hausdorff measure function to measure the sample path. This is the starting point for my joint work with Ciesielski [1] in 1961 which we will summarize in this lecture. We further describe some of the papers published in the last 40 years which built on the results and methods of [1], focusing only on those papers which find properties of the sample path of Brownian motion.
\end{abstract}

1. Introduction. It is a great pleasure for me to participate in this meeting to celebrate the work of Zbigniew Ciesielski. We first met in Cornell in 1960: both of us were young men, Zbigniew was 26 and I was 30. We both hoped to learn much from contact with the Polish probabilist, Mark Kac; we also learned from each other, particularly by our collaboration on [1], which is paper 13 in the list of Ciesielski publications. This paper provided precise answers to questions about the trajectory of Brownian motion in $\mathbb{R}^{d}$ $(d \geq 3)$. When I puzzled over the appropriate topic to talk about today, I realized that I had no unpublished results to be enthusiastic about, and then I recalled that the methods we developed in [1] had led to continued activity in the last 40 years, with hundreds of citations, including 5 in 2003. So I will pick out a subset of these developments and try to explain them.

Before embarking on this task, please allow me a few minutes to ponder on the nature of research in mathematics. We are fortunate in that when we discover a new theorem with a correct proof, we know that it will be true next year and for the following

2000 Mathematics Subject Classification: 60J65, 60G17, 60J55.

The author is Emeritus Professor of the University of Liverpool, and University of Virginia. The paper is in final form and no version of it will be published elsewhere. 
1000 years. Further, if it is a good theorem, it will become a building block for future research in ways which cannot be foreseen. This feature can only be explained by the nature of the universe in which we live and operate; it has a logical and self-consistent character. Mathematicians discover true theorems because they are there; we do not create truth. I remain convinced that there has to be a transcendent and personal God who designed and created the universe, including planet Earth, as a reflexion of his own logically consistent nature. As remarked by the astronomer, Sir James Jeans, "God must have been a mathematician".

So let us turn to the question of the trajectory of Brownian motion. The story starts with an English botanist called Brown who, in the mid-19th century, looked into his microscope at fine pollen suspended in water. He noticed very erratic movements by the pollen, which appeared to be moving in all directions. Mr Brown could not explain what he observed (for this was before any theory of molecules moving in a liquid) but, as a good scientist, he wrote down what he had observed. Around 1900, Einstein, among others, provided the explanation that the movement observed by Brown was caused by many thousands of collisions between the pollen and the molecules of the liquid; a French physicist, Jean Perrin, about 1910, used careful observations of this movement to estimate the diameter of a molecule (he got the right answer within a factor of 2). Since movements are the result of the net impetus from a large number of independent impulses from the bombardment of molecules, the central limit theorem leads to a reasonable hypothesis that the increment

$$
[X(t+h)-X(t)]
$$

should be normally distributed, with mean 0 and variance proportional to $h$, and that increments over disjoint time intervals should be independent. With this assumption, Wiener [2] (1923) proved the existence of a continuous random real function which is now called the Wiener process and became the mathematical model for 1-dimensional Brownian motion. Mathematical Brownian motion in $\mathbb{R}^{d}$ is the result of $d$ independent Wiener processes, one in each coordinate direction. This process has been studied extensively in the 20th century; 21 of Ciesielski's papers relate to this process or one of its close relatives. Much of the early work on the fine properties of Brownian motion was carried out by Paul Lévy [3] who clearly had an intuitive feel for the Brownian demon sitting on the path wondering what to do next. The development of Markov process theory including the understanding of the strong Markov property came later.

2. Properties established by 1960. By 1960 it was known that the Brownian motion process was transient for $d \geq 3$, recurrent but not point recurrent for $d=2$, and hits points for $d=1$.

Connections with classical potential theory had been established by Kakutani; these were used to prove that, for $d=2$, the path hits some points infinitely often though the trajectory has zero Lebesgue measure, for $d=3$ there are some double points but no points hit 3 times, and for $d \geq 4$ the path is simple. For all $d$, the sample path is everywhere continuous but nowhere differentiable, in fact the Wiener process has no points of increase. Local increments in time $h$ are of order $\sqrt{ } h$. In fact Lévy [3] had shown 
that there is an exact modulus of continuity

$$
\lim _{h \downarrow 0}\left[\sup _{0 \leq t \leq 1} \frac{|X(t+h)-X(t)|}{[2 h \log 1 / h]^{1 / 2}}\right]=1, \quad \text { with probability } 1 .
$$

This gives the maximum size of increments in small time; not much bigger than the typical result given by the law of the iterated logarithm, for fixed $t$

$$
\underset{h \downarrow 0}{\limsup } \frac{|X(t+h)-X(t)|}{[2 h \log \log 1 / h]^{1 / 2}}=1, \quad \text { almost surely. }
$$

By 1960, the Frostman theorems linking general potential theory with Hausdorff dimension had shown that, for $d \geq 2$, the trajectory $M(r)=\{x \in \mathbb{R}: x=X(t)$ some $t \in[0, r]$ has dimension 2 a.s., but the Frostman connections are not fine enough to establish the exact Hausdorff measure of $M(r)$. Recall the definition due to Hausdorff [4] (1919); given an increasing function $h:[0,1] \rightarrow \mathbb{R}$, with $h\left(0^{+}\right)=0$, and a subset $E \subset \mathbb{R}^{d}$, define

$$
\mathrm{h}-\mathrm{m}(E)=\lim _{\delta \downarrow 0} \inf \left[\sum_{i=1}^{\infty} h\left(\operatorname{diam} C_{i}\right): E \subset \bigcup_{i=1}^{\infty} C_{i} ; \operatorname{diam} C_{i}<\delta\right]
$$

Using (3) to prove that $\mathrm{h}-\mathrm{m}(E) \leq c$, we need to show that, for all $\delta, \epsilon>0$ we can find a cover $\bigcup C_{i} \supset E$ with $\operatorname{diam} C_{i}<\delta$ and $\sum h\left(\operatorname{diam} C_{i}\right)<c+\epsilon$; but to get the opposite inequality using (3) requires us to consider all small covers. In Hausdorff's paper [4] he needs 10 pages of careful analysis to prove this for the classical Cantor set and $h(s)=s^{\alpha}, \alpha=\log 2 / \log 3$. Paul Lévy [5] (1953) became interested in finding the right $h$-function to make h-m $(M(r))$ finite and positive; he used differential equation methods to show that, for $d \geq 3$,

$$
\mathrm{h}-\mathrm{m}(M(1))<c \text { a.s. with } h(s)=s^{2} \log \log (1 / s) .
$$

Our paper [1] had the main objective of proving that Lévy had the right $h$-function so that

$$
\mathrm{h}-\mathrm{m}(M(1)) \geq c>0 \text { a.s. with } h(s)=s^{2} \log \log (1 / s), \text { provided } d \geq 3 .
$$

A simple argument will then show the existence of finite positive constants $c_{d}, d \geq 3$ for which

$$
\mathrm{h}-\mathrm{m}(M(r))=c_{d} r \quad \text { for all } r \geq 0 \text { a.s. }
$$

3. Main idea. The key tool to use is a density theorem. Suppose $E \subset \mathbb{R}^{d}$ and there is a Borel measure $\mu$ concentrated on $E$ such that, if $B(x, r)$ denotes a ball centred at $x$ with radius $r$,

$$
\limsup _{r \downarrow 0} \frac{\mu B(x, r)}{h(2 r)} \leq \delta>0 \text { for all } x \text { in } E, \text { then h-m }(E) \geq \frac{1}{\delta} \mu(E) .
$$

The idea behind (4) is that when we cover $E$ by balls of radius $r_{i}$ small enough (note that any cover can be replaced by balls with centre in $E$ which are not much larger), then

$$
\sum h\left(2 r_{i}\right) \geq \frac{1}{\delta} \sum \mu B\left(x_{i}, r_{i}\right) \geq \frac{1}{\delta} \mu(E)
$$


The right choice for $\mu$ is a measure with closed support $M(1)$, which is spread uniformly in $M(1)$. We use the occupation measure

$$
\mu(A)=|\{0<t<1 ; X(t) \in A\}|
$$

(where $|E|$ denotes the Lebesgue measure of $E$ ). For $x=X(t)$, we can run the Brownian motion forwards and backwards from $t$ so that $\mu B(x, r)=T_{1}(r)+T_{2}(r)$, where

$$
T_{1}(r)=|\{s \in[0, t]: X(s) \in B(x, r)\}| \quad \text { and } \quad T_{2}(r)=|\{s \in[t, 1]: X(s) \in B(x, r)\}| .
$$

For $d \geq 3, X(t)$ is transient, so that for almost all $t$ in $[0,1], T_{1}, T_{2}$ are independent and have a distribution asymptotic as $r \downarrow 0$ to the total time spent in a ball of radius $r$ which we denote

$$
O_{d}(r)=|\{t>0:|X(t)|<r\}| \stackrel{\mathcal{D}}{=} r^{2} O_{d}(1), \quad \text { by scaling. }
$$

To apply (4), we only need asymptotic estimates for the large tail $\mathrm{P}\left\{O_{d}(1)>x\right\}$. In fact we can determine the exact distribution function for $O_{d}(r)$.

$$
\mathrm{P}\left\{O_{d}(r)>x\right\}=\sum_{s=1}^{\infty} \psi_{d, s} \exp \left(-\frac{p_{d, s}}{2 r^{2}} x\right),
$$

where $\left\{p_{d, s}\right\}$ are the positive zeros of the Bessel function $J_{k}(z)$ with order $k=\frac{1}{2} d-2$, and $\left\{\psi_{d, s}\right\}$ are known constants. Though the result is not needed for this paper, we also calculated the distribution of the first passage time $P_{d}(r)$ out of the ball of radius $r$. We were astonished to find that, for $d \geq 3$, the random variables $O_{d}(r)$ and $P_{d-2}(r)$ have identical distributions. Using a Borel-Cantelli argument, we now proved that, for fixed $t$, restarting the motion at $t$,

$$
\limsup _{r \downarrow 0} \frac{\mu B\left(X_{t}, r\right)}{h(2 r)} \leq 2 \limsup \frac{O_{d}(r)}{4 r^{2} \log \log 1 / r}=\frac{2}{p_{d, 1}^{2}} \quad \text { a.s. }
$$

and an application of Fubini with (4) now gives

$$
\mathrm{h}-\mathrm{m} X[0,1] \geq c_{d} p_{d, 1}^{2}>0 \quad \text { a.s. }
$$

4. Later developments. The techniques used in [1] have been modified and used many times in different situations. I can only outline a few of these. First let me mention the result for planar Brownian motion which required an additional idea due to Dan Ray [6] (1963). Planar Brownian motion is neighbourhood recurrent, but occupation measure can be defined as in (5). To get a handle on the distribution of $\mu B(x, r)$, Ray divides the time spent into excursions from $|x-z|=\frac{1}{2} r$ to $|x-z|=r$ and back to $|x-z|=\frac{1}{2} r$. The number $N(r)$ of these which happen before $t=1$ is a geometric random variable; the excursions are independent and during each excursion the time spent in $B(x, r)$ is a random variable whose expectation is of order $r^{2}$. As $r$ decreases to zero $N(r)$ increases and satisfies a law of the iterated logarithm. This allows Ray to prove that, for $x=X(t), 0<t<1$,

$$
\limsup _{r \downarrow 0} \frac{\mu B(x, r)}{\phi(r)}=\frac{1}{2} \text { a.s. }
$$

with $\phi(s)=s^{2} \log (1 / s) \log \log \log (1 / s)$. An application of (4) now yields $\phi-\mathrm{m}(M(1)) \geq$ $c>0$, as before. The density argument (4) has a converse which can be applied to the "good" points $x$ in $M(1)$ where (8) is true. But (8) is only true for $\mu$-almost all $x$ in 
$M(1)$. So to get a good upper bound we need to construct a cover for the bad points where (8) fails, and show that they do not contribute. This is accomplished in [7] (1964) to complete the proof that there is a positive constant $c$ such that $\phi-m M(r)=c r$ with $\phi(s)$ as in $(8)$.

Let us now look at the zero set $Z(t)=\{s \in[0, t]: X(s)=0\}$, where $X(t)$ is a Weiner process or 1-dimensional Brownian motion. Lévy had shown that there is a "local time" $L(s)$, determined by $X(t)$, which is non-decreasing, continuous and constant on the intervals complementary to $Z(t)$. Information obtained by Lévy implied that the Hausdorff dimension of $Z(t)$ is $\frac{1}{2}$ but $s^{1 / 2}-\mathrm{m}(Z(t))=0$. Paul Lévy asked for the correct measure function $f(s)$ to give $0<f-\mathrm{m}(Z(t))<\infty$ a.s. This problem was solved in a joint paper with Jim Wendel [8] (1966) by adapting the methods of [1] and using the observation that the zero set is stochastically the same as the range of a stable subordinator of index $\frac{1}{2}$; the jumps in the subordinator correspond to the complementary intervals of $Z$. It follows that there is a positive constant $c$ such that $f-\mathrm{m}(Z(t))=c L(t)$ for all $t>0$, where $f(s)=s^{1 / 2}(\log \log 1 / s)^{1 / 2}$. Hawkes [9] (1973) returns to the arguments in this paper to obtain accurate estimates for the constant $c_{\alpha}$ such that $g_{\alpha}-\mathrm{m} Y[0, s]=c_{\alpha} s$, where $g_{\alpha}(u)=u^{\alpha}(\log \log 1 / u)^{1-\alpha}$, and $Y(t)$ is a stable subordinator of index $\alpha, 0<\alpha<1$.

I will only mention two more examples out of the many cases where the methods of [1] produce the exact Hausdorff measure of a random set. Super-Brownian motion is a measure valued process which can be obtained as a limit of a critical branching process. Edwin Perkins [10] (1989) obtained the right Hausdorff measure function for the closed support $S\left(X_{t}\right)$ at time $t$ for $d \geq 3$; Perkins \& Le Gall [11] (1995) solved the same problem for $d=2$. The result is that, for $d \geq 3, h(s)=s^{2} \log \log 1 / s$; and if $d=2$, $h(s)=s^{2} \log 1 / s \log \log \log 1 / s$,

$$
X_{t}(A)=c_{d} \mathrm{~h}-\mathrm{m}\left(A \cap S\left(X_{t}\right)\right) \quad \text { for all Borel sets } A \text { and all } t \geq 0 \text { a.s. }
$$

The other example concerns the multiple points on the Brownian path. For $d=3$ the path has double points but no points hit more often. Jean-François Le Gall [10] (1986) uses "intersection local time" as a measure supported by the double points to show that the double points in $M(1)$ have finite positive Hausdorff measure for $h(s)=s[\log \log (1 / s)]^{2}$. He also solves the same probelm for the set of $k$-multiple points on a planar Brownian path.

5. Multifractal questions. Let me now try to answer the question: "Why is it possible to get a non-random answer when you calculate the exact Hausdorff measure of a random set such as the range $M(t)$ of a Brownian path in $\mathbb{R}^{d}(d \geq 2)$ ?" Essentially it is because only the points of typical behaviour contribute. Let me illustrate this by considering Brownian path variation. For subdivisions $(\pi)$ of $[0,1], 0=t_{0}<t_{1}<\cdots<t_{n}=1$, we define the mesh $\sigma(\pi)$ by $\sigma(\pi)=\max \left(t_{i}-t_{i-1}\right)$. Then

$$
V_{2}(X, \pi)=\sum_{i=1}^{n}\left[X\left(t_{i}\right)-X\left(t_{i-1}\right)\right]^{2}
$$

is the square variation. If $\pi_{k}$ denotes the diadic points $j 2^{-k}$ then a martingale argument shows that the square variation $V_{2}\left(X, \pi_{k}\right)$ converges almost surely to 1 as $k \rightarrow \infty$. How- 
ever, Lévy showed that $\max \left[V_{2}(X, \pi)\right]$ is unbounded as $\sigma(\pi) \rightarrow 0$. If $Q(\delta)$ denotes the set of partitions $\pi$ with $\sigma(\pi)<\delta$, and $\psi(s)=s^{2} / \log (1 / s)$, the uniform modulus (1) shows that $V_{\psi}(X, \pi)$ is bounded for all $\pi$. However $\psi$ is not the largest function with this property. I proved [12] (1973) that

$$
\lim _{\delta \downarrow 0}\left[\sup _{\pi \in Q(\delta)} V_{\phi}(X, \pi) t\right]=1, \quad \text { whenever } \quad \phi(s)=s^{2} / 2 \log \log (1 / s) .
$$

This $\phi$ is the asymptotic inverse for $[2 u \log \log (1 / u)]^{1 / 2}$ as $u \downarrow 0$. This means that even though the law of the iterated logarithm (2) is not valid for all $t$, you can not do any better when choosing a $\pi$ with small mesh. This is the reason why the arguments using upper asymptotic growth at a typical point yield the right answer in Hausdorff measure arguments, even though there are "bad points" where the growth rate is bigger. To try to quantify the bad points I collaborated with Steven Orey [13] (1974) to look at the set where (2) fails. We considered the set

$$
E(\alpha)=\left\{t \in[0,1]: \limsup _{h \downarrow 0} \frac{|X(t+h)-X(t)|}{[2 h \log 1 / h]^{1 / 2}}>\alpha\right\}, \quad \alpha>0 .
$$

By (1), $E(\alpha)$ is empty for $\alpha>1$, but we prove it is non-empty for $0 \leq \alpha \leq 1$, and its Hausdorff dimension $\operatorname{dim} E(\alpha)=1-\alpha^{2} . E(\alpha)$ contains "fast points" for the path, but it is not possible to choose $\pi$ with fine mesh to utilize these in calculating the variation.

This result is a precursor to the theory of multifractals developed in the physics literature in the 1980's as a means of analyzing special points in the support of a measure in $\mathbb{R}^{d}$. Consider the occupation measure defined by (5). It is easy to show that the local dimension

$$
d(\mu, x)=\lim \frac{\log \mu B(x, r)}{\log r}=2 \text { for all } x \text { in } M(1), \text { the support of } \mu ;
$$

so the spectrum of local dimension is trivial. The situation for the local time measure of a Wiener process is more interesting. We noted that $L(t)$ can be obtained as the occupation measure for a stable subordinator $Y$ of index $\frac{1}{2} . L(t)=\mu[0, t]$ where

$$
\mu(A)=|\{s \in[0,1]: Y(s) \in A\}| .
$$

At a typical point $x$ of $Z$, the zero set of Wiener process, we have

$$
d(\mu, x)=\lim \frac{\log \mu(x-r, x+r)}{\log r}=\frac{1}{2},
$$

but this is not true for all $x$ in $Z$. The spectrum is considered in joint work with Xiaoyu $\mathrm{Hu}[15]$ (1995), where we prove that

$$
d(\mu, x)=\liminf _{r \downarrow 0} \frac{\log \mu(x-r, x+r)}{\log r}=\frac{1}{2}
$$

for all $x$ in $Z$, but if

$$
A_{\beta}=\left\{x \in Z: \limsup _{r \downarrow 0} \frac{\log \mu(x-r, x+r)}{\log r}=\beta\right\},
$$

then $A_{\beta}$ is non-empty with $\operatorname{dim} A_{\beta}=\frac{1}{2 \beta}-\frac{1}{2}$ for $\frac{1}{2} \leq \beta \leq 1$. This should be interpreted as meaning there are no dimension-thick points in $Z$, but there is a spectrum of dimensionthin points. As in [14], we can obtain a spectrum of the thick points by introducing a 
logarithmic term. This is done in joint work with Narn-Reuih Shieh [16] (1998), where we show that

$$
\operatorname{dim} Q_{\beta}=\frac{1}{2}\left(1-\beta^{2}\right), \quad \text { where } \quad Q_{\beta}=\left\{x \in Z: \limsup _{r \downarrow 0} \frac{\mu(x-r, x+r)}{[r \log 1 / r]^{1 / 2}}=\beta\right\} .
$$

In a recent series of papers, Dembo, Peres, Rosen and Zeitouni [20] (2000-2002) the authors obtain a spectrum for both thick points and thin points on a Brownian path by introducing a logarithmic factor. For example, thick points on the path for $d \geq 3$ are given by

$$
\operatorname{dim}\left\{x \in \mathbb{R}^{d}: \limsup \frac{\mu B\left(X_{t}, r\right)}{r^{2} \log 1 / r}=a\right\}=2-\frac{a q_{d}^{2}}{2},
$$

where $q_{d}$ is the first positive zero of the Bessel function $J_{k}(z)$, with $k=\frac{1}{2} d-2$; so the set is empty is $a>4 / q_{d}^{2}$. (This uses the precise form of the tail of $O_{d}(r)$, found in [1].)

The situation for super-Brownian motion is also interesting; again there are no dimension-thick points in the spectrum, but there are dimension-thin points. In joint work with Ed Perkins [17] (1998), we show that, a.s.,

$$
\liminf _{r \downarrow 0} \frac{\log X_{t} B(x, r)}{\log r}=2
$$

for all $x$ in the support $S\left(X_{t}\right)$, but

$$
\operatorname{dim}\left\{x \in \mathbb{R}^{d}: \limsup _{r \downarrow 0} \frac{\log X_{t} B(x, r)}{\log r}=a\right\}=\frac{8}{a}-2, \quad \text { for } 2 \leq a \leq 4 .
$$

We note that in both [15] and [18] the multifractal formalism used in the physics literature for finding the dimension spectrum breaks down completely.

These kinds of investigations continue. J. Blath \& Peter Morters [19] (2004) introduce a logarithmic factor to investigate thick points for super-Brownian motion, and W. Konig with Peter Morters [20] (2002) investigate thick points for Brownian intersection local times using the structure considered by Le Gall in [10]. That is (more than) enough for today. One wonders what further developments there may be in the next 40 years, but most of us will not be alive to learn that.

\section{References}

[1] Z. Ciesielski and S. J. Taylor, First passage times and sojourn times for Brownian motion and the exact Hausdorff measure of the sample path, Trans. Amer. Math. Soc. 103 (1963), $434-450 .^{1}$

[2] N. Wiener, Differential space, Jour. Math. Physics 2 (1923), 131-171.

[3] P. Lévy, Processus Stochastiques et mouvements Browniens, Gauthier-Villars, Paris, 1948.

[4] F. Hausdorff, Dimension und ausseres, Mass, Math. Annalen 79 (1919), 157-179.

[5] P. Lévy, La mesure de Hausdorff de la courbe du mouvement Brownien, Gior. Inst. Italien Attuari 16 (1953), 273-302.

[6] D. Ray, Sojourn times and the exact Hausdorff measure of the sample path of planar Brownian motion, Trans. Amer. Math. Soc. 106 (1963), 436-444.

\footnotetext{
${ }^{1}$ Note that this paper is No. 13 in the list of publications by Z. Ciesielski.
} 
[7] S. J. Taylor, The exact Hausdorff measure of the sample path of planar Brownian motion, Proc. Camb. Phil. Soc. 60 (1964), 250-258.

[8] S. J. Taylor and J. G. Wendel, The exact Hausdorff measure of the zero set of a stable process, Zeit. für Wahrscheinlichkeitstheorie 6 (1966) 170-180.

[9] J. Hawkes, The measure of the range of a subordinator, Bull. Lon. Math. Soc. 5 (1973), $21-28$.

[10] J.-F. Le Gall, the exact Hausdorff measure of Brownian multiple points I and II, Seminar on stochastic processes 1986, 107-137, and 1988, 193-197.

[11] E. A. Perkins, The Hausdorff measure of the closed support of super-Brownian motion, Ann. Inst. H. Poincaré 25 (1989), 205-224.

[12] J.-F. Le Gall and E. A. Perkins, The Hausdorff measure of the support of two-dimensional super-Brownian motion, Annals of Prob. 23 (1995) 1719-1747.

[13] S. J. Taylor, Exact asymptotic estimates of Brownian path variation, Duke Math. Jour. 29 (1972), 219-241.

[14] S. Orey and S. J. Taylor, How often on a Brownian path does the law of iterated logarithm fail?, Proc. Lon. Math. Soc. 28 (1974)174-193.

[15] X. Hu and S. J. Taylor, The multifractal structure of stable occupation measure, Stoch. Proc. and Appl. 66 (1997), 283-299.

[16] N.-R. Shieh and ac S. J. Taylor, Logarithmic multifractal spectrum of stable occupation measure, Stoch. Proc. and Appl. 75 (1998), 249-261.

[17] E. A. Perkins and S. J. Taylor, The multifractal structure of super-Brownian motion, Ann. Inst. H. Poincaré 34 (1998), 97-138.

[18] J. Blath and P. Morters, Thick points of super-Brownian motion, Prob. Th. and Related Fields, to appear.

[19] W. Konig and P. Morters, Brownian intersection local times: upper tail asymptotics and thick points, Annals of Prob. 30 (2002), 1605-1636.

[20] A. Dembo, Y. Peres, J. Rosen and O. Zeitouni, Thick points for spatial Brownian motion, Annals of Prob. 28 (2000), 1-35; Thin points for Brownian motion, Annals Inst. H. Poincaré 36 (2000), 749-774; Thick points for planar Brownian motion and the ErdosTaylor conjecture on random walk, Acta Math. 186 (2001), 239-270; Thick points for intersections of planar Brownian paths, Trans. Amer. Math. Soc. 364 (2002), 4969-5003. 